University of New Orleans

ScholarWorks@UNO

$7-1980$

\title{
Inversion of the nonlinear equations of reflection ellipsometry for uniaxial crystals in symmetrical orientations
}

M. Elshazly-Zaghloul

R. M.A. Azzam

University of New Orleans, razzam@uno.edu

Follow this and additional works at: https://scholarworks.uno.edu/ee_facpubs

Part of the Electrical and Electronics Commons, and the Physics Commons

\section{Recommended Citation}

M. Elshazly-Zaghloul and R. M. A. Azzam, "Inversion of the nonlinear equations of reflection ellipsometry for uniaxial crystals in symmetrical orientations," J. Opt. Soc. Am. 70, 880-883 (1980)

This Article is brought to you for free and open access by the Department of Electrical Engineering at ScholarWorks@UNO. It has been accepted for inclusion in Electrical Engineering Faculty Publications by an authorized administrator of ScholarWorks@UNO. For more information, please contact scholarworks@uno.edu. 
Jr., and E. Wolf are gratefully acknowledged. This work was supported in part by NSF Grants SER77-06914 and ENG79-08038.

${ }^{1}$ Gouy, C. R. Acad. Sci. Paris 110, 1251-1253 (1890).

${ }^{2}$ Gouy, Ann. Chim. Phys. 6, XXIV, 145-213 (1891).

3P. Debye, Ann. Phys. 30, 755 (1909). A discussion of Debye's work can be found in J. Picht, Optische Abbildung (Vieweg \& Sohn, Braunschweig, 1931). References to much of the early work in this field, including that of F. Reiche and K. Schwarzschild, can also be found in this book.

${ }^{4} \mathrm{~A}$. Rubinowicz, "On the anomalous propagation of phase in the focus," Phys. Rev. 54, 931-936 (1938).

${ }^{5} \mathrm{E}$. H. Linfoot and E. Wolf, "Phase Distribution near Focus in an Aberration-free Diffraction Image," Proc. Phys. Soc. London 69, 823-832 (1956).

${ }^{6}$ Due to an algebraic error, the original paper states that the wavelength is decreased by this factor (E. Wolf, private communication).

${ }^{7}$ H. Kogelnik and T. Li, "Laser Beams and Resonators," Appl. Opt. 5, 1550-1567 (1966).

${ }^{8}$ P. F. Byrd and M. D. Friedman, Handbook of Elliptic Integrals for Engineers and Physicists (Springer-Verlag, Berlin, 1954), p. 3.

${ }^{9} \mathrm{M}$. Abramowitz and I. A. Segen, Handbook of Mathematical Functions (Dover, New York, 1965), Eqs. (17.3.11) and (17.3.12).

${ }^{10}$ N. N. Lebedev, Special Functions and their Applications (Dover, New York, 1972), p. 88.

\title{
Inversion of the nonlinear equations of reflection ellipsometry for uniaxial crystals in symmetrical orientations
}

\author{
M. Elshazly-Zaghloul \\ Electrical Engineering Department, Faculty of Engineering, Cairo University, Cairo, Egypt \\ R. M. A. Azzam \\ Department of Electrical Engineering, School of Engineering, University of New Orleans, Lakefront, New Orleans, Louisiana 70122 \\ (Received 15 June 1979; revised 7 January 1980)
}

\begin{abstract}
The complex ordinary $\left(N_{o}\right)$ and extraordinary $\left(N_{e}\right)$ refractive indices of an absorbing uniaxial crystal can be determined using reflection ellipsometry. The measurements are taken with the optic axis parallel and perpendicular to the crystal's surface. The equations obtained are solved without resort to iterative methods; $N_{o}$ and $N_{\mathrm{e}}$ are determined separately. Sixteen solution sets $\left(N_{o}, N_{e}\right)$ are obtained and the correct solution can be easily identified. We present an optimum angle of incidence that minimizes the relative errors in $N_{o}$ and $N_{e}$.
\end{abstract}

\section{INTRODUCTION}

With the increased interest in uniaxial crystals and their applications, e.g., in electro-optics, the need arises for accurate determination of their optical properties. One of the powerful tools for this purpose is ellipsometry. By studying the change of the polarization state of light upon reflection from the surface of a crystal, the optical properties of the crystal can be determined.

Until now the equations relating the optical properties of a uniaxial crystal and the quantities measured by a conventional ellipsometer were solved using iterative numerical methods. In this paper we present a new inversion method for determining separately the ordinary $\left(N_{o}\right)$ and extraordinary $\left(N_{e}\right)$ refractive indices. An eighth-degree polynomial in $N_{o}$ is first obtained whose coefficients are determined from the measured data. This polynomial is solved using a new numerical method that is not iterative in nature. ${ }^{1}$ Knowing the solution for $N_{o}$, we obtain $N_{e}$ by direct substitution. ${ }^{2}$ A detailed example in which this method is applied to a $\mathrm{GaSe}$ crystal is presented. Also, using a simulated error analysis, we determine the range of angles of incidence $\phi$ with the least percent error in $N_{o}$ and $N_{e}$ caused by an error of $\phi$ for ice, calcite, and GaSe crystals.

\section{METHOD}

For a uniaxially symmetric crystal, two relatively simple cases can be distinguished depending on whether the optic axis (axis of symmetry) is in the plane of incidence or perpendicular to it. ${ }^{3-5}$ With respect to the first case, consider only the configuration where the optic axis is perpendicular to the reflecting crystal surface. We take the optic axis parallel to the $z$ axis of a Cartesian $x y z$ coordinate system. The optical constants $N_{o}\left(N_{x}\right)$ and $N_{e}\left(N_{z}\right)$ of the crystal may then be written as

$$
\begin{gathered}
N_{o}=N_{x}=N_{y}=n_{o}-j k_{o}, \\
N_{e}=N_{z}=n_{e}-j k_{e} .
\end{gathered}
$$

When the $z$ axis is perpendicular to the crystal surface, the Fresnel reflection coefficients take the form

$$
\begin{gathered}
r_{p}=\left(N_{e} N_{o} \cos \phi-n X_{e}\right) /\left(N_{e} N_{o} \cos \phi+n X_{e}\right), \\
r_{s}=\left(n \cos \phi-X_{o}\right) /\left(n \cos \phi+X_{o}\right),
\end{gathered}
$$

where $\phi$ is the angle of incidence, $n$ is the refractive index of the ambient, and

$$
X_{e}=\left(N_{\theta}^{2}-n^{2} \sin ^{2} \phi\right)^{1 / 2},
$$




\begin{tabular}{|c|c|c|c|c|}
\hline Solution No. & $N_{o 1}$ & $N_{o 2}$ & $N_{e 1}$ & $N_{e 2}$ \\
\hline 1 & $0.940-j 0.151 \times 10^{-4}$ & $-0.940+j 0.151 \times 10^{-4}$ & $1.052-j 0.0684$ & $-1.052+j 0.0684$ \\
\hline 2 & $1.0+j 0.0$ & $-1.0+j 0.0$ & $1.0+j 0.0$ & $-1.0+j 0.0$ \\
\hline 3 & $1.0+j 0.0$ & $-1.0+j 0.0$ & $1.0+j 0.0$ & $-1.0+j 0.0$ \\
\hline 4 & $0.611-j 0.147$ & $-0.611+j 0.147$ & $0.957+j 0.648 \times 10^{-2}$ & $-0.957-j 0.648 \times 10^{-2}$ \\
\hline 5 & $0.686+j 0.418$ & $-0.686-j 0.418$ & $0.949+j 0.0145$ & $-0.949-j 0.0145$ \\
\hline 6 & $1.743-j 0.479$ & $-1.743-j 0.479$ & $0.990+j 0.104 \times 10^{-2}$ & $-0.990-j 0.104 \times 10^{-2}$ \\
\hline 7 & $4.260-j 0.617$ & $-4.260+j 0.617$ & $1.199+j 1.959$ & $-1.199-j 1.959$ \\
\hline 8 & $3.766-j 0.906$ & $-3.766+j 0.906$ & $2.797-j 0.469$ & $-2.797+j 0.469$ \\
\hline
\end{tabular}

$$
X_{o}=\left(N_{o}^{2}-n^{2} \sin ^{2} \phi\right)^{1 / 2} .
$$

If the $z$ axis (optic axis) is in the crystal surface and is perpendicular to the plane of incidence, the Fresnel reflection coefficients become

$$
\begin{gathered}
r_{p}=\left(N_{o}^{2} \cos \phi-n X_{o}\right) /\left(N_{o}^{2} \cos \phi+n X_{o}\right), \\
r_{s}=\left(n \cos \phi-X_{e}\right) /\left(n \cos \phi+X_{e}\right),
\end{gathered}
$$

where $n, \phi, X_{o}$, and $X_{e}$ have the same meaning as before.

From Eqs. (2), we can write

$$
\begin{gathered}
\rho_{1}=r_{p} / r_{s} \\
=\frac{N_{e} N_{o} \cos \phi-n X_{e}}{N_{e} N_{o} \cos \phi+n X_{e}} \frac{n \cos \phi+X_{o}}{n \cos \phi-X_{o}} .
\end{gathered}
$$

This equation can be rewritten as

$$
\frac{K_{1} n \cos \phi-X_{o}}{-n \cos \phi+K_{1} X_{o}}=\frac{N_{e} N_{o} \cos \phi}{n X_{e}},
$$

where

$$
K_{1}=\left(\rho_{1}+1\right) /\left(\rho_{1}-1\right) .
$$

Similarly, from Eqs. (4), we have

$$
\rho_{2}=\frac{N_{o}^{2} \cos \phi-n X_{o}}{N_{o}^{2} \cos \phi+n X_{o}} \frac{n \cos \phi+X_{e}}{n \cos \phi-X_{e}},
$$

which can be rewritten as

$$
\frac{K_{2} N_{o}^{2} \cos \phi+n X_{o}}{N_{o}^{2} \cos \phi+K_{2} n X_{o}}=\frac{n \cos \phi}{X_{e}},
$$

where

$$
K_{2}=\left(\rho_{2}+1\right) /\left(\rho_{2}-1\right) .
$$

Substituting the value of $X_{e}$ from Eq. (8a) into Eq. (6a) and squaring both sides, we obtain

$$
N_{e}^{2} N_{o}^{2}=n\left(\frac{K_{1} n \cos \phi-X_{o}}{-n \cos \phi+K_{1} X_{o}}\right)\left(\frac{N_{o}^{2} \cos \phi+K_{2} n X_{o}}{K_{2} N_{o}^{2} \cos \phi+n X_{o}}\right) .
$$

From Eqs. (3), we can write

$$
\begin{aligned}
& N_{e}= \pm\left(X_{e}^{2}+n^{2} \sin ^{2} \phi\right)^{1 / 2}, \\
& N_{o}= \pm\left(X_{o}^{2}+n^{2} \sin ^{2} \phi\right)^{1 / 2} .
\end{aligned}
$$

Substituting Eqs. (10) and the value of $X_{e}$ from Eq. (8a) into Eq. (9) and rearranging, we obtain an eighth-degree polynomial in $X_{o}$ in the form;

$$
\sum_{n=0}^{8} M_{n} X_{o}^{n}=0
$$

where

$$
\begin{gathered}
M_{0}=D^{\prime} C^{\prime} E_{1}-F^{\prime} E^{\prime} E, \\
M_{1}=D^{\prime}\left(-B^{\prime} E_{1}+C^{\prime} D_{1}\right)-F^{\prime}\left(-B^{\prime} E+E^{\prime} D\right), \\
M_{2}=C^{\prime} E_{1}+D^{\prime}\left(A^{\prime} E_{1}-B^{\prime} D_{1}+C^{\prime} C_{1}\right) \\
-F^{\prime}\left(E-B^{\prime} D+E^{\prime} C\right), \\
M_{3}=-B^{\prime} E_{1}+C^{\prime} D_{1}+D^{\prime}\left(A^{\prime} D_{1}-B^{\prime} C_{1}+C^{\prime} B_{1}\right) \\
-F^{\prime}\left(D-B^{\prime} C+E^{\prime} B\right), \\
M_{4}=A^{\prime} E_{1}-B^{\prime} D_{1}+C^{\prime} C_{1}+D^{\prime}\left(A^{\prime} C_{1}-B^{\prime} B_{1}+C^{\prime} A_{1}\right) \\
-F^{\prime}\left(C-B^{\prime} B+E^{\prime} A\right), \\
M_{5}=A^{\prime} D_{1}-B^{\prime} C_{1}+C^{\prime} B_{1}+D^{\prime}\left(A^{\prime} B_{1}-B^{\prime} A_{1}\right) \\
-F^{\prime}\left(B-B^{\prime} A\right), \\
M_{6}=A^{\prime} C_{1}-B^{\prime} B_{1}+C^{\prime} A_{1}+D^{\prime} A A_{1}-F^{\prime} A, \\
M_{7}=A^{\prime} B_{1}-B^{\prime} A_{1}, \\
M_{8}=A^{\prime} A_{1},
\end{gathered}
$$

and

$$
\begin{gathered}
A=\cos ^{2} \phi, \\
B=2 n K_{2} \cos \phi, \\
C=n^{2}\left(K_{2}^{2}+2 \sin ^{2} \phi \cos ^{2} \phi\right), \\
D=2 n^{3} K_{2} \cos \phi \sin ^{2} \phi, \\
E=n^{4} \sin ^{4} \phi \cos ^{2} \phi, \\
A_{1}=n^{2} \cos ^{2} \phi\left(\cos ^{2} \phi+K_{2}^{2} \sin ^{2} \phi\right), \\
B_{1}=2 n^{3} K_{2} \cos \phi, \\
C_{1}=n^{4}\left[K_{2}^{2} \cos ^{2} \phi+\sin ^{2} \phi+2 \sin ^{2} \phi \cos ^{2} \phi\left(\cos ^{2} \phi\right.\right. \\
E_{1}=n^{6} \sin ^{4} \phi \cos ^{2} \phi\left(\cos ^{2} \phi+K_{2}^{2} \sin ^{2} \phi\right), \\
A^{\prime}=K_{1}^{2}, \\
B^{\prime}=2 n K_{1} \cos ^{2} \phi, \\
C^{\prime}=n^{2} \cos ^{2} \phi, \\
D^{\prime}=n^{2} \sin ^{2} \phi, \\
E^{\prime}=n^{2} K_{1}^{2} \cos ^{2} \phi, \\
F^{\prime}=n^{4} .
\end{gathered}
$$




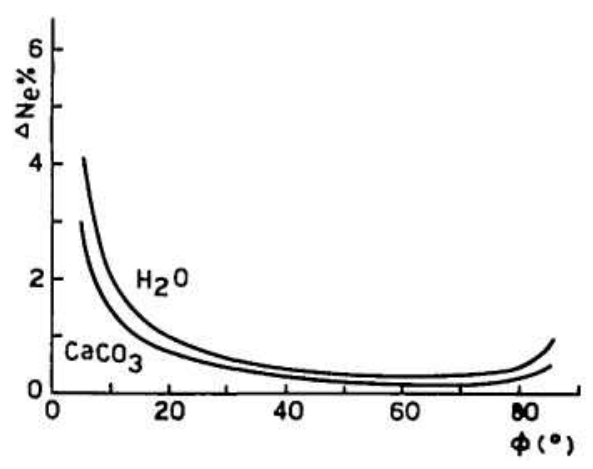

FIG. 1. Percent error of $N_{\theta}$ for ice and calcite crystals as a function of $\phi$ $\left({ }^{\circ}\right)$ caused by an angle-of-incidence error $\delta \phi=+0.1^{\circ}$.

Note, from Eqs. (12) and (13), that the polynomial coefficients $M_{n}$ of Eq. (11) are all functions of the known $(n, \phi)$ and measured $\left(K_{1}, K_{2}\right)$ quantities. Using a readily available computer program, Eq. (11) can easily be solved for $X_{0}{ }^{1}$ Accordingly, we obtain eight values for $X_{0}$. For each value of $X_{o}$, we obtain one value for $X_{e}$ by direct substitution in Eq. (8a). Now we have eight pairs of solutions $\left(X_{o}, X_{e}\right)$. From Eqs. (10) we obtain four $\left(N_{o}, N_{e}\right)$ solution pairs for each $\left(X_{o}, X_{e}\right)$ pair. In general, most of these 32 solution pairs for $\left(N_{o}, N_{e}\right)$ can be readily rejected by applying the physical constraint of a complex refractive index with a positive real part and a negative imaginary one. The unrejected solution pairs can be further screened on the basis of a reflectance measurement. ${ }^{6}$ The correct solution pair can easily be distinguished using such a method.

\section{NUMERICAL EXAMPLE}

We have checked the above scheme by using the published values of $N_{o}$ and $N_{\rho}$ for several uniaxial crystals ${ }^{7,8}$ in forward calculations, Eqs. (5)-(7), to get the ratios of reflection coefficients $\rho_{1}$ and $\rho_{2}$ at selected values of $\phi$. The inversion scheme of Sec. I, Eqs. (8)-(11), was applied using $\rho_{1}, \rho_{2}$, and $\phi$ (in backward calculations) to determine the solution sets $\left(N_{o}, N_{e}\right)$.

We take as a detailed example the case of a GaSe crystal at a wavelength of $0.3 \mu$ at $\phi=70^{\circ}$ and assuming $n=1$ (i.e., an air ambient). The coefficients of the eighth-degree polynomial, Eq. (11), obtained from direct calculations are given by

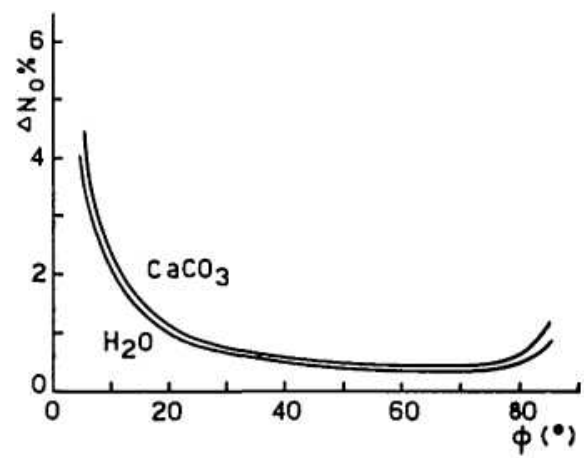

FIG. 2. Percent error of $N_{o}$ for ice and calcite crystals as a function of $\phi$ $\left({ }^{\circ}\right)$ caused by an angle-of-incidence error $\delta \phi=+0.1^{\circ}$.

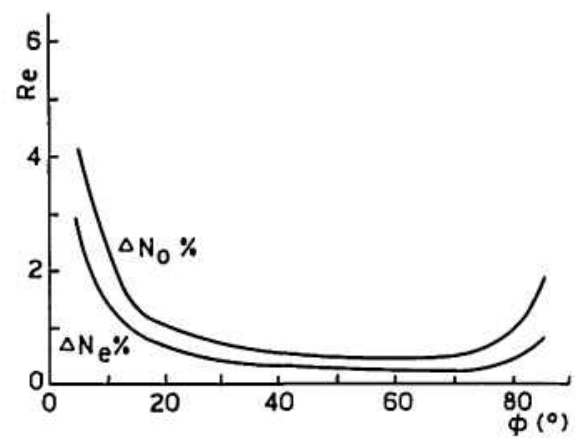

FIG. 3. Percent error of the real part of $N_{o}$ and $N_{\theta}$ in the case of a GaSe crystal as a function of $\phi\left(^{\circ}\right)$ caused by an angle-of-incidence error $\delta \phi$ $=+0.1^{\circ}$.

$$
\begin{gathered}
M_{0}=(0.108-j 0.291) \times 10^{-3}, \\
M_{1}=(-5.08+j 0.418) \times 10^{-2}, \\
M_{2}=(2.72+j 0.899) \times 10^{-2}, \\
M_{3}=(3.73-j 0.351) \times 10^{-1}, \\
M_{4}=(-2.53-j 0.739) \times 10^{-1}, \\
M_{5}=(53.8+j 0.819) \times 10^{-2}, \\
M_{6}=(0.106+j 0.154), \\
M_{7}=(-0.125-j 0.121), \\
M_{8}=(0.157+j 0.199) \times 10^{-1} .
\end{gathered}
$$

The eight solutions of the above obtained polynomial are thus

$$
\begin{gathered}
X_{01}=(0.257-j 0.533) \times 10^{-2}, \\
X_{02}=(0.342+j 0.000), \\
X_{03}=(-0.342+j 0.000), \\
X_{04}=(0.121-j 0.739), \\
X_{05}=(0.341+j 0.839), \\
X_{06}=(-1.496+j 0.559), \\
X_{07}=(4.157-j 0.652), \\
X_{08}=(3.653-j 0.934) .
\end{gathered}
$$

Table I gives the solutions obtained for $N_{o}$ and $N_{e}$. From this

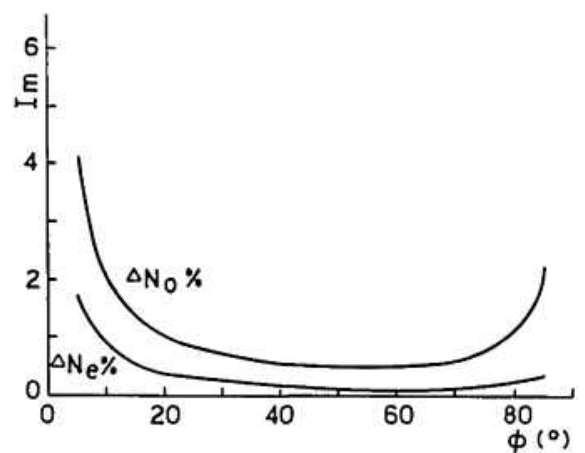

FIG. 4. Percent error of the imaginary part of $N_{o}$ and $N_{\theta}$ in the case of a GaSe crystal as a function of $\phi\left({ }^{\circ}\right)$ caused by an angle-of-incidence error $\delta \phi=+0.1^{\circ}$. 
table, it is clear that the correct solution is easily identified, solution $8 ; N_{o}=(3.766,-0.906)$ and $N_{e}=(2.797,-0.469)$.

\section{ERROR ANALYSIS}

Forward calculations combined with the inversion method explained in Sec. I have been used to determine the effect of errors of the angle of incidence $\phi$ on the derived ordinary and extraordinary refractive indices as obtained from the conventional ellipsometric measurements of $\rho_{1}$ and $\rho_{2}$. Figures 1-4 show the percent error of $N_{o}$ and $N_{e}$ as a function of $\phi$ caused by $0.1^{\circ}$ error of $\phi$ in the cases of ice, calcite, and GaSe crystals. From these figures the conclusion can be drawn that the choice of $\phi$ in the range from $50-70^{\circ}$ leads to the minimum percent errors of the derived optical properties $\left(N_{o}\right.$ and $\left.N_{e}\right)$ arising from given errors of angles of incidence. ${ }^{9}$

${ }^{1}$ A computer program based on the Automated Taylor Series method of Dr. Chang of the Computer Department of the University of Nebraska-Lincoln was used. It yields highly accurate results in a very short time (better than 12 significant figures). The program is in FORTRAN IV and can be obtained by writing to Dr. Chang or to the authors. The closeness of $N_{o}$ and $N_{e}$ should cause no problem because they are separated in two polynomials.

${ }^{2}$ The suggested inversion method is superior to the previously used numerical methods in several aspects. First, it does not require previous knowledge of the optical properties to be measured. Of course this is essential whenever we have an unknown crystal to be characterized. Second, its accuracy does not depend on the starting values of $N_{o}$ and $N_{e}$ (it needs no starting values). Third, it separates the determination of $N_{o}$ and $N_{e}$.

${ }^{3}$ R. A. W. Graves, "Determination of the optical constants of anisotropic crystals," J. Opt. Soc. Am. 59, 1225-1228 (1969).

${ }^{4} \mathrm{~A}$. Wünsche, "Neue formeln für die reflexion und brechung des lichtes an anisotropen medien," Ann. Phys. (Leipzig) 25, 201-214 (1970).

${ }^{5}$ D. den Engelsen, "Transmission ellipsometry and polarization spectrometry of thin layers," J. Phys. Chem. 76, 3390-3397 (1972).

${ }^{6}$ M. Elshazly-Zaghloul, R. M. A. Azzam, and N. M. Bashara, "Explicit solution for the optical properties of a uniaxial crystal in generalized ellipsometry," in Proceedings of the Third International Conference on Ellipsometry, edited by N. M. Bashara and R. M. A. Azzam (North-Holland, Amsterdam, 1977), pp. 281-292.

${ }^{7}$ F. Meyer, E. E. de Kluizenaar, and D. den Engelsen, "Ellipsometric determination of the optical anisotropy of gallium selenide," J. Opt. Soc. Am. 63, 529-552 (1973).

${ }^{8} \mathrm{~J}$. M. Bennett and H. E. Bennett, "Polarization," in Handbook of Optics, edited by W. G. Driscoll and W. Vaughan (McGraw-Hill, New York, 1978), pp. 10-1 to 10-164.

${ }^{9}$ This range of $\phi$ for minimum percent error of $N_{o}$ and $N_{e}$ (caused by $0.1^{\circ}$ error of $\phi$ ) is the same as we have previously found ${ }^{6}$ in the case of generalized ellipsometry applied to uniaxial crystals with the optic axis in the plane of the surface but is neither parallel nor perpendicular to the plane of incidence. 